Rapport - Société canadienne d'histoire de l'Église catholique

\title{
Le contrat d'association des Jésuites avec Poutrincourt
}

\section{Lucien Campeau}

Volume 27, 1960

URI : https://id.erudit.org/iderudit/1007400ar

DOI : https://doi.org/10.7202/1007400ar

Aller au sommaire du numéro

Éditeur(s)

La Société canadienne d'histoire de l'Église catholique

ISSN

0318-6148 (imprimé)

1927-7075 (numérique)

Découvrir la revue

Citer cet article

Campeau, L. (1960). Le contrat d'association des Jésuites avec Poutrincourt. Rapport - Société canadienne d'histoire de l'Église catholique, 27, 13-21.

https://doi.org/10.7202/1007400ar

Tous droits réservés @ La Société canadienne d'histoire de l'Église catholique, 1961
Ce document est protégé par la loi sur le droit d'auteur. L'utilisation des services d'Érudit (y compris la reproduction) est assujettie à sa politique d'utilisation que vous pouvez consulter en ligne.

https://apropos.erudit.org/fr/usagers/politique-dutilisation/ 


\section{Le contrat d'association des Jésuites avec Poutrincourt}

On sait que, le 20 janvier 1611, Charles de Biencourt, fils du sieur de Poutrincourt, et Thomas Robin, son comparse, d'une part, les PP. Pierre Biard et Enemond Massé, futurs missionnaires d'Acadie, d'autre part, signaient devant notaires un contrat qui faisait des missionnaires des associés au commerce de la Nouvelle-France et les bénéficiaires d'une moitié des revenus dans le voyage qui allait s'engager. La minute de cet acte semble aujourd'hui perdue, mais le texte en a été imprimé à trois reprises, entre 1613 et $1618^{1}$, comme preuve de la rapacité des Jésuites. Les ennemis des Pères les accuseront de s'être intéressés davantage au commerce qu'à la conversion des Indiens. Les Biencourt leur feront écho, ainsi que Lescarbot. Ils montreront les missionnaires appliqués à s'assurer le contrôle de Port-Royal, propriété de Poutrincourt, et ne sauront jamais aucun gré à la marquise de Guercheville, qui avait fourni les fonds nécessaires à cette association, de sa libéralité. On dira que les Jésuites ont mis les aumônes qu'ils avaient reçues à usure nautique, c'est-à-dire à un intérêt qui demeurait suspect aux moralistes du temps, mais qui était d'un usage courant dans le commerce maritime. Enfin, Lescarbot attribuera constamment aux Jésuites le dommageable retard occasionné par les tractations préalables au contrat.

Voyons d'abord quelle est la substance de cet acte. Dans l'ensemble, c'est une reconnaissance par Biencourt des obligations qu'il a contractées envers les Jésuites, beaucoup plus qu'une convention bilatérale. Voici les dispositions distribuées par articles.

$1^{\circ}$ Biencourt, Thomas Robin et les deux Jésuites sont d'accord sur la valeur totale de la cargaison du navire, qui s'élève à 7,600 livres tournois.

$2^{\circ}$ Biencourt et Thomas Robin reconnaissent avoir reçu des Jésuites 3,800 livres tournois, soit la moitié du prix de la cargaison; en conséquence, ils les associent à leur entreprise et leur promettent la moitié des profits qui en proviendront, une fois les frais défalqués.

1 Une première publication eut lieu sous ce titre: Contrat d'association des Jésuites au trafique de Canada, pour apprendre à Paul de Gimont, l'un des donateurs d'advis pour les Jésuites contre le Recteur et Université de Paris, et à ses semblables pourquoy les Jésuites sont depuis peu arrivez en Canada (s. 1., 1613). L'année suivante, le contrat était reproduit par le Factum du procez entre messire Jean de Biencourt, chevalier, sieur de Poutrincourt, baron de Sainct-Just, appelant, d'une part, et Pierre Biard, Enemond Massé et consorts, soy disans prestres de la Société de Jésus, intimez (s. 1., 1614). Lescarbot le réimprimait aussi dans la troisième édition de son Histoire de la Nouvelle-France... (Paris, Adrian Périer, 1618), pp. 665-667. Ces ouvrages sont utilisés comme sources de notre étude. 
$3^{\circ}$ Biencourt et Robin confessent également que les Jésuites leur ont prêté la somme de 737 livres en pur et loyal prêt, sans aucun intérêt mentionné, et ils s'engagent à la remettre à leur retour.

$4^{\circ}$ Biencourt a encore emprunté des Jésuites et de Thomas Robin la somme de 1,225 livres, pour le radoub de son navire, et il promet de la restituer au retour.

$5^{\circ}$ Les Jésuites et Thomas Robin paieront à Biencourt un fret de 1,000 livres, qui pourra être déduit des 1,225 livres qui leur sont dues.

La facture de ce contrat est tout à fait insolite et reflète une inexpérience et une imprudence qui ne sont pas habituelles en ce genre de documents. On pourrait en faire ici une longue et fastidieuse discussion. Mais je ne dois pas abuser du privilège que j'ai d'ennuyer mes auditeurs.

Je dirai donc seulement l'essentiel. Tout d'abord, le rôle de Robin, dans cette affaire, ne peut être que fictif. On sait qu'il n'avait pas de capitaux ${ }^{2}$, et l'imprécision, absolument étrange, qui entoure sa participation à l'entreprise fait de lui un membre purement nominal. Les Biencourt eux-mêmes, que l'on voit rendre des comptes aux Jésuites, ne considéront jamais Robin comme un associé ${ }^{3}$. Nous suivrons ici leur exemple.

En second lieu, le contrat estime la valeur de la cargaison à 7,600 livres. Au contraire, toutes les autres sources, tant celles qui proviennent des Jésuites que celles qui émanent des Biencourt et de Lescarbot ${ }^{4}$, s'accordent à dire que cette cargaison ne se montait pas à plus de 4,000 livres et se trouvait entièrement couverte par la contribution que fit madame de Guercheville pour le compte des Pères. On sait par ailleurs que Biencourt était sans argent, qu'il a même dû emprunter des missionnaires la somme de 1,962 livres pour payer les dépenses étrangères à la cargaison. Enfin, si Biencourt avait eu en main les 3,800 livres que le contrat estime comme sa part du prix du chargement, il aurait eu un capital plus considérable que ne possédera jamais son père en aucun de ses voyages ${ }^{5}$. Il eût donc pu financer seul l'entreprise et on ne le verrait pas dépendre entièrement des armateurs et obligé d'emmener avec lui les Jésuites qu'il détestait. Il n'est pas risqué d'affirmer que le contrat a doublé fictivement la valeur de la cargaison. déjà entièrement payée par les 3,800 livres que les Jésuites ont procurées. Le procédé, en soi, n'était pas malhonnête et il pouvait se justifier par la nécessité de

2 Pierre Biard, Relation de la Nouvelle-France (Lyon, 1616), 126-127; réédit. Thwaites, The Jesuit Relations and allied Documents, 3 (1897): 164. Cette Relation, d'après la réédition de Thwaites, est la source principale de l'étude qui va suivre, accompagnée d'autres documents portant sur la même mission et publiés dans les quatre premiers volumes de cette collection.

3 Charles de Biencourt à son père, Port-Royal, 13 mars 1612, dans Factum du procez, p. 28.

4 Documentation sur ce détail, dans Thwaites, $J R, 1: 126-136,140-144 ;$ 2: 212-214, 174-176; Factum du procez, 6-7.

5 Poutrincourt fit un embarquement avec 1,800 livres en 1611, un autre avec 1,500 livres en 1612 , et un troisième en 1613 , avec 3,116 livres. 
créer à Biencourt un titre égal à celui des Pères en vue du partage des profits par moitié.

Ce sont les circonstances qui font de cet acte une transaction tout à fait inégale et inique. Biencourt ne fournit que son navire; il ne fait dans l'affaire aucun autre déboursé. Sa moitié de la cargaison est payée par les Jésuites. De plus, ces derniers lui paieront un louage de 1,000 livres pour le transport dans son vaisseau de leur moitié de la charge. C'est là un fret élevé, qui n'est pas loin d'égaler la valeur du navire lui-même. Ajoutons que Biencourt a obtenu des Pères 1,962 livres en prêt pur et simple, sans aucun intérêt. C'est un avantage absolument inespéré en ce genre d'entreprise et qu'il n'eût jamais pu attendre d'autres que de missionnaires désintéressés ${ }^{6}$. Plus grave encore était cette circonstance que les Pères participaient aussi bien aux frais qu'aux profits. Or les profits ne pouvaient leur échoir qu'au titre du commerce. Mais les frais, eux, couvraient les dépenses de deux entreprises, l'une commerciale, l'autre colonisatrice. Si le commerce profitait aux deux parties, la colonie, qui exigeait la plus forte partie des déboursés, en salaires et en matériel, rapportait exclusivement aux Biencourt et les missionnaires n'y acquéraient aucun avantage. Le plus probable, et ce qui est même certain d'après l'impression générale laissée par les sources, c'est que les dépenses de la colonie ont englouti, et au-delà, le maigre profit qu'on pouvait retirer du commerce. Il ne semble pas que les Pères aient jamais récupéré, sinon en négligeable partie, les capitaux qu'ils avaient mis dans l'affaire.

Pour tout dire en quelques mots, mises à part les dépenses partagées également entre les contractants, Biencourt n'a au départ qu'à amortir une obligation de 962 livres due aux Jésuites ${ }^{7}$, alors que ceux-ci doivent commencer sur leur moitié des revenus par éteindre une mise de 4,800 livres ${ }^{8}$ avant de faire un denier de profit. En outre, toute la moitié des frais de la colonie que les Jésuites soldent sans en rien retirer demeure une valeur acquise aux Biencourt. On voit dès lors si ces derniers ont eu raison de se plaindre de ce contrat et de cette association et si l'accusation de rapine imputée aux Jésuites peut raisonnablement se soutenir.

Le contrat ainsi brièvement discuté, faisons rapidement son histoire. On sait que Poutrincourt, accompagné de son fils Biencourt, avait repris en 1610 la colonisation de Port-Royal en Acadie, poste qui avait été abandonné par De Monts en 1607. Il avait amené avec lui un prêtre, qui, le 24 juin 1610, fit 21 baptêmes d'Indiens, sans avoir eu le loisir de les préparer et de les instruire. Poutrincourt lui-même avait conçu le plan de cette évangélisation-éclair afin d'obtenir des ressources de la

- De tels prêts, pour une expédition en Nouvelle-France, ne se faisaient que pour de petites sommes, cinquante, quatre-vingts ou cent livres, et l'intérêt consistait en quarante pour cent du profit rapporté par le capital prêté.

7 Biencourt devait 1,962 livres aux Jésuites, mais ceux-ci s'étaient obligés à payer un fret de mille livres en retour.

8 Les 3,800 livres qui représentent la valeur de leur mise, plus 1,000 livres de fret. 
Cour. C'est pour retirer les avantages de cette bonne action qu'il renvoya, dès juillet 1610, son fils en France.

Lorsque Biencourt débarqua à Dieppe, le 21 août 1610, sa situation financière ne devait pas être brillante. Son père avait épuisé ses ressources dans le voyage précédent. Le vaisseau n'avait été que trois semaines en Acadie. Les Indiens avaient été presque tous invisibles. D'autres marchands avaient devancé Poutrincourt. La récolte des fourrures ne pouvait donc qu'être maigre, d'autant plus qu'il n'y avait pas sur l'Álianiique de cenires conme Taudoussac ou Quévec, où les Indiens affluaient jusque du milieu du continent. Le meilleur du revenu dut être celui de la pêche, que Biencourt fit pendant quinze jours sur le banc de Terre-Neuve. C'était peu pour payer les salaires et les autres dépenses, comme pour solder les dettes laissées au début de l'année chez les armateurs. Il fallait en plus redouber un navire vieilli et abîmé, le charger, payer les frais du séjour en France, les démarches à la Cour, etc.

En arrivant à Dieppe, Biencourt alla trouver deux armateurs calvinistes, un nommé Dujardin et un autre, appelé Abraham Duquesne. Poutrincourt avait probablement pris langue avec eux avant son départ. Biencourt leur demanda de radouber son navire et de le charger. Que pouvait-il offrir en retour? Des espérances. Il s'en allait à la Cour, où l'annonce des baptêmes faits récemment provoquerait sans doute la générosité de la Reine et des grands. Du même coup, on obtiendrait aussi le monopole du commerce, qui obligerait tous les armateurs à offrir à Poutrincourt leur argent pour profiter de son privilège. Y a-t-il eu, dès ce moment, une association entre les marchands et Biencourt, comme celui-ci l'a affirmé aux Jésuites? Il n'en existe aucune preuve, et Biencourt a déclaré lui-même qu'il n'y avait pas de contrat entre lui et les calvinistes. Il n'apparaît aucune trace de rupture d'association dans le contrat que nous étudions, où il devait pourtant s'en trouver. Les Jésuites ne se sont pas substitués aux marchands; ils sont simplement associés à Biencourt. Dans les circonstances, des commerçants aussi avisés que Duquesne et Dujardin ne pouvaient traiter avec le fils de Poutrincourt qu'à des conditions extrêmement désavantageuses pour lui ${ }^{9}$. Et encore, il leur était plus profitable de louer un bon navire, uniquement destiné au commerce, et de l'envoyer en Nouvelle-France, la traite des fourrures restant toujours libre. Ils consentaient sans doute à appareiller le navire de Biencourt contre argent comptant, mais une association ne pouvait leur sourire aux conditions que Biencourt leur

9 Les marchands ont naturellement en vue le profit, et non une cuvre de bienfaisance. Ils ne sont donc disposés qu'à financer une expédition rigoureusement commerciale. Biencourt, privé des 5,762 livres que lui apporteront les Jésuites, reste avec son seul navire, qui a besoin, aux termes du contrat, d'un radoub de 1,225 livres. En supposant qu'il loue son vaisseau aux marchands, il n'en peut retirer que 2,000 livres, toujours d'après l'estime du contrat. Sur ces 2,000 livres, il doit dépenser 1,225 livres en radoub. Il lui reste 775 livres pour entretenir pendant un an la colonie de Port-Royal, où il faut ravitailler une vingtaine d'hommes, payer leurs salaires, sans compter le matériel nécessaire à la colonisation. Ces 775 livres qui sont tout ce que Biencourt peut attendre de son voyage dans les circonstances, sont loin de suffire à payer seulement les salaires. 
proposait. Encore ne se pressent-ils pas de commencer l'appareillage. Quand les Jésuites arriveront à Dieppe à la fin d'octobre, rien n'aura encore été fait dans ce sens. N'était-il pas sage de savoir si Biencourt pourrait payer?

Ce dernier se rend donc à Paris, à la fin d'août 1610, et commence les démarches pour obtenir des subsides de la Cour, assisté de son ami Lescarbot. Le bruit se répand dans la capitale que l'évangélisation de la Nouvelle-France a été commencée par Poutrincourt. Le P. Coton, confesseur du Roi, l'apprend et s'étonne, à mesure que les jours passent, que Biencourt ne l'aille pas visiter pour inviter enfin les Jésuites au voyage, selon la promesse de son père. Madame de Guercheville, épouse du duc de Liancourt et première dame d'honneur de la Reine, s'informe auprès du Jésuite des progrès de l'affaire des missions et celui-ci lui fait part de sa surprise.

C'est ici qu'entre en scène Thomas Robin, qu'on nomme aussi sieur de Coloignes. C'est un jeune homme un peu plus âgé que Biencourt, d'une famille bien cotée dans la noblesse parisienne, mais qui n'a pas encore atteint la majorité légale. Il paraît avoir eu plus de liant et d'éloquence persuasive que de scrupules. Compagnon de Poutrincourt au voyage précédent, il est revenu en France avec le fils Biencourt, que son père, confiant peut-être dans la faconde et les relations du jeune Parisien, semble avoir confié aux soins de ce dernier. Peut-être même Robin était-il mandaté comme agent de Poutrincourt. Madame de Guercheville, alertée par le P. Coton, fait venir Robin et lui demande quelles sont les intentions de Poutrincourt par rapport aux Jésuites. Robin répond que le chef de Port-Royal lui a confié toute la charge de l'embarquement, que lui-même est associé pour cinq ans au gentilhomme, auquel il s'est engagé à fournir tout ce dont aurait besoin la colonie pendant ce temps. Sans doute, il n'a reçu aucune instruction particulière concernant les Jésuites, mais il est certain que Poutrincourt se sentirait honoré de les avoir auprès de lui. Pour leur entretien, lui-même est prêt à s'en charger, comme il fait pour le reste de la dépense. Beau discours, mais qui ne devait pas coûter beaucoup à un jeune aventurier sans le sou. La grande dame l'assura que les missionnaires ne lui seraient pas à charge, puisque le Roi défraierait leurs dépenses.

Elle envoie De Coloignes au provincial des Jésuites, le P. Christophe Baltazar, qui reçoit les mêmes assurances. Sur ce, le Provincial écrit au P. Biard, qui se morfondait alors à Poitiers, de venir en hâte à Paris, où il dut arriver sur la fin de septembre. On lui adjoignit comme compagnon de voyage le $\mathrm{P}$. Enemond Massé, résidant alors à Paris comme assistant du P. Coton à la Cour.

Que pensait Biencourt des avances que Robin avait faites aux Jésuites? Il avait contre ceux-ci toutes les préventions de son père. Son ami Lescarbot, rempli des sentiments des milieux parlementaires à l'égard de la Compagnie, l'exhortait à ne pas se laisser prendre. Sur ces entrefaites, arrive, le $1^{\text {er }}$ octobre, la date longtemps attendue de l'audience royale. Biencourt s'y présente avec Lescarbot, ce dernier armé d'un rapport imprimé, qu'il présente à la Reine. Marie de Médecis 
remercie le jeune voyageur, le félicite et l'assure de sa bienveillance; puis elle lui dit sans phrases que deux Jésuites vont partir avec lui. Il n'y avait guère à répondre à l'expression d'une volonté aussi déterminée. Pourtant, Lescarbot va tenter un dernier effort, sans doute avec l'assentiment de Biencourt. Il reprend la plume et allonge son rapport du double, fait imprimer en hâte sa prose fraîche qu'il ajoute à la précédente, met en tête une préface dédicatoire à la Reine, afin de présenter l'œuvre complétée à l'audience d'adieu que la Régente réserve à Biencourt dans quelques jours ${ }^{10}$. On n'avait pas besoin en NouvelleFrance, écrivait Lescarbot, de ces "docteurs subilmes", qui seraient mieux employés en France à combattre les hérétiques. Et il ajoutait: " Joint qu'il y a certaine sorte de gens desquels on ne se peut pas bien asseurer, faisans métier de censurer tout ce qui ne vient pas à leur maximes et voulans commander par tout ${ }^{11}$. "L'audience eut lieu à Monceaux, le 7 octobre. Le résultat n'en fut pas heureux, au sens de Lescarbot. Il espérait que Biencourt en sortirait les bras chargés d'étrennes pour les nouveaux baptisés, avec, dans sa poche, un rouleau dûment scellé et authentiqué contenant un monopole du commerce; or Biencourt n'avait sur les bras que deux Jésuites.

Biencourt se retrouvait donc sur les pavés de la capitale aussi pauvre qu'auparavant, et davantage encore à cause des dépenses encourues durant le séjour à Paris. A défaut des cadeaux de la Reine, il pensa qu'il serait à propos d'obtenir une charte de vice-amiral, avec laquelle il entendait lever des droits sur les navires qui viendraient commercer en Nouvelle-France. C'était encore des frais en perspectıve. C'est ici que les avances prématurées de Thomas Robin pouvaient être mises à profit. Les Jésuites avaient obtenu comme viatique de la Reine 1,500 livres. Si l'on ne pouvait se passer des Jésuites, aussi bien profiter d'eux. C'est peut-être à l'occasion de la visite que lui firent les PP. Biard et Massé que Biencourt obtint d'eux un premier prêt de 737 livres. En retour, il leur donna rendez-vous à Dieppe pour le 27 octobre.

Le fils de Poutrincourt, accompagné de Robin, reprend le chemin de Dieppe, vers le milieu du mois d'octobre. Le courage des deux jeunes hommes est à la baisse. Le voyage de Paris a été raté. Ils vont affronter de nouveau les marchands et ils sont plus démunis qu'ils n'ont jamais été, puisqu'ils n'ont même plus d'espoirs à offrir. Ce qui va suivre a toutes les apparences d'un scénario admirablement monté et magistralement mené, qui a pu naître dans l'imagination fertile de Robin. En tout cas, celui-ci y paraît comme le metteur en scène.

S'il n'était pas dans l'intérêt de Duquesne et de Dujardin de s'associer avec Biencourt au temps de son débarquement, que penser de leurs dispositions après la déconfiture du jeune homme? Sans doute, les marchands ne refuseraient pas de remplir leur engagement pour l'appareillage du navire, mais pourvu qu'ils soient assurés d'être payés

10 Ce rapport imprimé en deux fois est connu sous le nom de La Conversion des sauvages qui ont esté baptizés en la Nouvelle-France... (Paris, [1610] ). On le trouve dans Thwaites, JR, 1: [49]-113.

11 Thwaites, $J R, 1: 80$. 
avant le départ. Justement, c'est une assurance que Biencourt ne peut donner. Car les 737 livres prêtées par les Jésuites semblent bien s'être écoulées par d'autres fissures. Et puis, cette somme ne répond pas du tout aux besoins. Tout va se passer maintenant comme si un plan avait été concerté entre Biencourt et les armateurs calvinistes: les attitudes vont être exagérées, dramatisées, les effets ménagés et contrôlés vers une issue prédéterminée, comme dans une pièce de théâtre. Tout est si bien calculé que cela ne peut être l'effet du hasard: le but, on le devine, est d'utiliser les Jésuites pour se procurer l'argent qui assurera aux marchands un bon bénéfice et mettra Biencourt en chemin.

Vers le 27 octobre 1610, les PP. Biard et Massé arrivent à Dieppe, disposés à prendre la mer. On leur avait promis que tout serait prêt à cette date. Mais le radoub et le chargement sont à peine commencés, depuis des mois que le vaisseau est au port. Le bruit de l'arrivée des Jésuites s'est déjà repandu dans la ville. Les deux armateurs calvinistes s'en vont trouver Biencourt et Robin et jouent la première scène de la petite comédie. Ils s'étonnent et s'indignent de ce que les Jésuites vont être du voyage. Ils n'armeront pas un vaisseau pour transporter ces pestes en Nouvelle-France. Ils retirent leur parole et ne feront rien tant que les Jésuites n'auront pas été écartés. Biencourt et Robin répondent qu'ils ne sont pas libres d'accepter ou de refuser les missionnaires; c'est la Reine qui a ordonné leur passage. D'ailleurs, les Jésuites ne coûteront rien aux marchands, puisque la souveraine elle-même se charge de leurs frais. Les calvinistes sont inébranlables. Que l'on choisisse entre eux et les Jésuites !

On va raconter cette scène aux deux Pères, qui sont atterrés. Le $P$. Biard, toujours ardent, demande s'il n'y a pas moyen de forcer les calvinistes à remplir leur contrat. Robin répond vertueusement que de tels contrats ne se mettent pas par écrit entre nobles, et que par conséquent on ne peut traîner les marchands en justice. Assertion évidemment fausse, puisqu'on possède encore des centaines de ces contrats, signés par des nobles, et même par le Roi. Les Français, nobles ou non, aimaient bien trop le papier ! En réalité, il n'existait ni contrat ni association, mais une simple entente de fournisseur à client, malgré ce qu'on voulait laisser croire aux Jésuites pour leur tenir la dragée haute. Le $\mathrm{P}$. Biard se contente donc de la réponse.

Est-ce en ce moment de dépression que les deux Pères consentent à Biencourt un prêt de 1,225 livres ${ }^{12}$, destiné, leur dit-on, au radoub ? On ne saurait l'assurer, mais le moment était sûrement propice. Quant à l'entreprise, on ne trouve pas d'autre moyen pour la dépanner que d'envoyer Robin en messager auprès de la Reine. Peut-être fourniraitelle les moyens de contourner l'obstacle ? Si Robin attendait de l'argent, il se faisait illusion. C'est un lettre qu'il rapporta, adressée au gouverneur de Dieppe, le sieur de Sigongnes. Celui-ci, aussitôt, assemble

12 Le radoub du navire, au témoignage du P. Biard, était compris dans les services des marchands calvinistes, et donc aussi dans la somme de 3,800 livres qui leur a été payée. Ce chiffre de 1,225 livres est évidemment trop fort pour le radoub d'un vaisseau qui n'en valait peut-être pas autant. A quoi Biencourt a-t-il fait servir cet argent? 
le consistoire de la communauté calviniste et l'exhorte à intervenir auprès des deux coreligionnaires. Ils refusent de céder. Que l'on saisisse leurs biens, si l'on veut; ils ne souffriront pas un seul Jésuite sur un navire chargé par eux. Qu'on leur envoie toutes sortes d'autres robes, capucins, récollets, cordeliers, ils les traverseront volontiers; mais des Jésuites, non. Les marchands ne dédaignent même pas de s'amuser: si la Reine, disent-ils, est prête à expatrier en Nouvelle-France tous les Jésuites de l'ancienne, à la bonne heure! Ils offrent à fréter le navire et à en faire les frais; pas autrement. Marie de Médecis, informée, juge indigne de s'abaisser à solliciter des marchands; elle décide que les missionnaires passeront une autre fois. Magnifique occasion pour Biencourt de se passer des Jésuites, s'il n'avait eu d'eux un besoin si urgent, juste à ce moment.

Nous touchons le sommet du drame. Les deux missionnaires, devenus la risée publique ${ }^{13}$, doivent se retirer au collège $d^{\prime} \mathrm{Eu}$, leur plus proche maison. Mais les acteurs ont conscience d'avoir bien rempli leurs rôles. Les marchands sont désormais confiants de rentrer dans leurs frais. "Aussitôt, ils mirent dans le navire marchandises et toutes autres choses en grande abondance ${ }^{14}$," écrit le P. Biard. Biencourt de son côté, promet aux Jésuites qu'il ne partira pas sans eux. Car ces derniers n'ont pas renoncé au voyage, malgré l'abandon de la Reine. A Eu, ils se procurent une relique de saint Laurent, qu'ils doivent emporter. A Rouen, on leur fait des aumônes et ils attendent toujours que le navire soit paré. Biencourt et Robin poursuivent l'œuvre si bien commencée. Le second repart vers Paris, où il va trouver madame de Guercheville. A la marquise qui s'indigne de l'outrecuidance des marchands, il suggère qu'il y a bien un moyen de passer les Jésuites en Nouvelle-France. Ce serait de dédommager les marchands, pour libérer le navire de leur emprise. Tous les services qu'ils ont pu rendre seraient bien couverts par la somme de 4,000 livres, si on pouvait se la procurer. La grande dame est une femme d'action. Aussitôt, elle parcourt le cercle de ses connaissances, les princes, les ducs, tous les nobles de la Cour. Elle a tôt fait d'amasser la somme, qu'elle met entre les mains de Robin. Celui-ci l'emporte à Dieppe, où l'argent servira à solder les frais des deux marchands. Tout le monde pourra être content: les marchands qui auront été payés; Biencourt qui verra son navire appareillé; les Jésuites qui vont s'embarquer.

Madame de Guercheville avait montré à l'égard de Biencourt une insigne libéralité. Mais elle n'entendait pas que les missionnaires, en considération desquels elle agissait ainsi, ne pussent en tirer profit. Marie de Médecis leur avait bien octroyé une subvention de 1,500 livres, déjà plus qu'engloutie dans les prêts consentis à Biencourt; mais ce n'étaient pas les 2,000 livres promises par Henri IV. En outre, on n'était pas certain que ce présent deviendrait annuel, comme l'avait

13 David Asseline, Les Antiquitez et chroniques de la ville de Dieppe (2 vol., Dieppe-Paris-Rouen, 1874), 2: 154-155.

14 «Nam illico et merces et omnia imposuerunt in navim quam abundantissime» (Archivum romanum Societatis Iesu, Gal. 109 I, f. 7). 
encore promis le feu Roi. Les Jésuites ne pouvaient vivre sans revenus, si on voulait les rendre indépendants de la colonie, comme le demandait leur caractère. Robin vantait la rentabilité du commerce canadien; la marquise pensa que sa contribution pourrait devenir un fonds capable d'établir solidement l'avenir de la mission. Le capital initial pourrait être réinvesti dans les voyages suivants et les profits qu'il rapporterait feraient fonction d'une rente suffisante aux besoins de la mission. Elle mit donc comme condition à sa générosité que les Jésuites deviendraient associés au commerce et en retireraient la moitié des bénéfices. Ce fut la raison du contrat d'association.

Dans ce travail, nous avons usé, sans négliger aucune bribe d'information disponible, du privilège qu'a l'historien de pouvoir reconstituer les faits. Il en était besoin, à cause des incohérences et des obscurités nombreuses de la documentation. Certains faits essentiels nous ont paru évidents. Les marchands calvinistes ont certainement profité, et considérablement, de l'intervention de la marquise ${ }^{15}$, et il est non moins certain qu'une association avec Biencourt ne pouvait leur être avantageuse. Il n'est donc pas injurieux pour eux de penser qu'ils ont désiré et voulu que les Jésuites servissent à leur procurer un profit qu'ils ne pouvaient obtenir sans eux. Biencourt, également, a tiré le plus grand avantage de ce contrat. Avant comme après l'association, il est clair qu'il détestait et craignait les Jésuites ${ }^{16}$. Mais il était sans ressources et les Pères étaient, à ce moment, la dernière qui lui restât. De là à supposer qu'il y a eu entente entre Biencourt et les marchands pour arriver justement au résultat obtenu, il n'y a qu'un pas. Une telle hypothèse, à son tour, illumine un tas d'obscurités et de contradictions, qui resteraient incompréhensibles sans elle. Cette histoire du contrat ne peut se comprendre, si on ne suppose qu'elle a été une comédie jouée aux Jésuites pour les dépouiller.

\author{
Lucien Campeau, s.j. \\ Professeur d'Histoire de l'Église \\ à la Faculté de Théologie de \\ IImmaculée-Conception de Montréal.
}

15 Si l'on compare le prix de cette cargaison avec les dépenses qui se faisaient habituellement en des vaisseaux de cette catégorie, le montant de 3,800 livres est exceptionnellement élevé.

16 Poutrincourt, jusque-là, avait tout fait pour éviter les Jésuites. Déjà à l'automne de 16l1, Biencourt manifeste sa méfiance et son hostilité aux deux missionnaires. 